\title{
Influência da parasitemia na evolução da doença de Chagas crônica
}

\author{
The influence of the parasitemia on the evolution of the \\ chronic Chagas' disease
}

\author{
Cleudson Castro ${ }^{1}$, Aluízio Prata ${ }^{2}$ e Vanize Macêdo ${ }^{1}$
}

\begin{abstract}
RESUMO
Estudou-se clínica e parasitologicamente, durante 13 anos, 190 indivíduos com infecção chagásica objetivando investigar a relação entre parasitemia ea evolução da doença de Chagas crônica. Comparou-se a parasitemia de 56 indivíduos xenopositivos e 134 xenonegativos, em 1988/91 com a evolução clínica encontrado-se 22 (39,3\%) e 50 (37,3\%), respectivamente, com evolução progressiva. Estratificou-se a parasitemia em 1988/91, em alta, média e baixa e a correlação clínica mostrou que 5 (62,5\%) , 10 (41,7\%) e 57 (36.1\%) indivíduos, respectivamente, apresentaram evolução progressiva, sem diferença estatística significante, $(p>0,05)$. No período de 1976/91, houve 20 pacientes com parasitemia constante e 59 sem parasitemia, observandose evolução progressiva em $6(30 \%)$ e 17 (28,8\%), respectivamente. Houve seis pacientes com alta parasitemia e, 59 sem parasitemia, verificado-se que $3(50 \%)$ e 17 (28,8\%), respectivamente, apresentaram evolução progressiva, sem diferença estatística significante, $(p>0,05)$. As médias das idades daqueles com alta, média e baixa parasitemias foram 39,6; 45,3 e 41,5 anos, respectivamente, $(p>0,05)$. As médias das idades dos pacientes com evolução progressiva, inalterada e regressiva foram respectivamente, 46,4; 39,8 e 32,6 anos, com diferença estatística significante entre aqueles com evolução progressiva e regressiva, $(p<0,05)$. Sugere-se que a alta parasitemia não influenciou na evolução da doença crônica.
\end{abstract}

Palavras-chaves: Parasitemia. Doença de Chagas. Evolução clínica. Projeto Mambaí. Trypanosoma cruzi. Tripanosomíase americana. Xenodiagnóstico.

\begin{abstract}
During 13 years, 190 individuals with chagasic infection were submitted to clinical and parasitological examinations to investigate the relationship between parasitemia and the evolution of chronic chagasic infection. Fifty-six patients with positive xenodiagnosis and 134 with negative exams were compared from 1988 to 91, it was found that 22 (39.3\%) and $50(37.3 \%)$, respectively, presented disease progression. The parasitemia was stratified into high, medium and low and the relation with the disease evolution showed that $5(62.5 \%), 10(41.7 \%)$ and 57 (36.1\%), respectively, presented progressive disease, though without a statistically significant difference $(p>0.05)$. When 20 patients with persistent parasitemia in 1976/91, were compared with 59 with negative xenodiagnosis, a progressive evolution was observed in 6 (30\%) and 17 (28.8\%), respectively. Comparing six patients with high parasitemia and 59 with negative exams, it was verified that $3(50 \%)$ and 17 (28.8\%), respectively, showed progressive disease, but this was not statistically significant, $(\mathrm{p}>0.05)$. Mean age with high, medium and low parasitemia were 39.6, 45.3 and 41.5 years, respectively, $(p>0.05)$. Mean age in patients showing progressive, unaltered and regressive evolution was 46.4, 39.8 and 32.6 years, respectively, with a statistically significant difference between progressive and regressive evolution $(p<0.05)$. It is suggested that high parasitemia did not have an influence on the evolution of the chronic infection.
\end{abstract}

Key-words: Parasitemia. Chagas' disease. Clinical evolution. Mambaí project. Trypanosoma cruzi. American tripanosomiasis. Xenodiagnosis.

\footnotetext{
1. Núcleo de Medicina Tropical da Universidade de Brasília, Brasília DF, Brasil. 2. Disciplina de Doenças Infecciosas e Parasitárias da Faculdade de Medicina do Triângulo Mineiro, Uberaba MG.

Endereço para correspondência: Prof. Cleudson Castro. Núcleo de Medicina Tropical/UnB. Caixa Postal 04517, 70919-970 Brasília DF, Brasil.

Fax 55 61 273-2811.

e-mail: tropical@unb.br

Recebido para publicação em 13/5/2004

Aceito em 17/9/2004
} 
A importância do parasita como agente patogênico na forma crônica da doença de Chagas tomou grande impulso nas últimas décadas. Recentemente foram descobertos e estudados especialmente por pesquisadores do Triângulo Mineiro 2345673032 , sítios onde aparentemente 0 Trypanosoma cruzi se oculta. 0 T. cruzi tem seu habitat no interior da célula e na infecção crônica multiplica-se lentamente. Após algum tempo rompe-se a membrana celular sendo lançados na corrente sanguínea novos parasitos que aí permanecem algum tempo para a seguir penetrar e albergar em outras células. Em geral o parasitismo tecidual é baixo na fase crônica. Eventualmente, encontra-se alto parasitismo mormente na supra-renal sendo poucas as descrições destes casos ${ }^{16}{ }^{31}$, restringindo-se a pacientes autopsiados e exaustivamente estudados. Sabemos muito pouco sobre 0 alto parasitismo tecidual no chagásico crônico em vida. A informação disponível é a parasitemia que permite fazer conjecturas sobre sua importância na evolução clínica da doença. 0 presente trabalho tem como objetivo estudar a correlação dos vários níveis de parasitemia ao longo de 13 anos, com a evolução clínica da doença de Chagas na fase crônica, tema repleto de interrogações.

\section{PACIENTES E MÉTODOS}

Dentre os cerca de 1.000 indivíduos com a forma crônica da doença de Chagas do Projeto Mambaí (Goiás, Brasil), há uma grupo de 190 pacientes que tem sido estudado do ponto de vista parasitológico, clínico, eletrocardiográfico e radiológico, sendo possível fazer a correlação clínica parasitológica. Todos foram diagnosticados para doença de Chagas pela imunofluorescência indireta, hemaglutinação passiva e fixação do complemento, realizadas em dois laboratórios de referência. Dentre os 190, 173 apresentaram seis reações positivas, 15 mostraram três a cinco exames positivos e um negativo e dois apresentaram três exames positivos e três negativos, porém com xenodiagnósticos positivos.

0 estudo longitudinal da parasitemia foi feito através de quatro xenodiagnósticos clássicos, realizados entre os anos de 1976 e 1991, em média 13 anos de estudo. Todos os pacientes fizeram o xenodiagnóstico (xeno) no início, no interstício e no final do período. Cada exame foi realizado com 40 triatomíneos da espécie Dipetalogaster maximus. Para semiquantificar a parasitemia de cada indivíduo usou-se a classificação de Castro ${ }^{8}$, que levou em consideração o percentual de pools positivos em relação aos examinados. Os pacientes foram classificados em baixa, média e alta parasitemias quando zero a 19,9\%, 20 a $67,9 \%$ e $68 \%$ ou mais, respectivamente, do total de pools examinados foram positivos.

0 exame clínico foi realizado pelo mesmo observador em 1975/76, 1980/82 e 1986/91 e constou de um interrogatório dirigido para 0 sistema cardiovascular, aparelho digestivo, especialmente sobre obstipação e sistema nervoso. № exame físico, anotou-se 0 pulso, a tensão arterial e, no exame cardiovascular a posição do ictus, ritmo cardíaco, características das bulhas, sopros, frêmitos e sinais de descompensação cardíaca. No exame do abdome, buscou-se palpar fecaloma. № exame neurológico pesquisou-se motricidade, coordenação, sinal de Romberg e o reflexo aquileu. Concomitante ao exame clínico em cada período, foi feito o eletrocardiograma (ECG) e a abreugrafia do esôfago. 0 ECG constou de 12 derivações com quatro complexos em cada uma e D2 de $20 \mathrm{~cm}$. Os traçados dos três períodos foram interpretados pelo mesmo observador segundo os critérios da American Heart Association adaptandose 0 código de Minesota para alterações de Q, ST e T. 0 estudo radiológico contrastado do esôfago foi realizado com aparelho de abreugrafia adaptado para filme de $70 \mathrm{~mm}$. 0 paciente foi colocado em posição oblíqua anterior direita sendo feita uma abreugrafia após a ingestão de $75 \mathrm{ml}$ de solução baritada e outra 60 segundos após. A interpretação dos esofagogramas foi feita em três períodos distintos pelo mesmo observador, segundo os critérios de Rezende et al ${ }^{26}$. Cada indivíduo foi diagnosticado nos diversos períodos, em uma das seguintes formas da doença de Chagas crônica: indeterminada, cardíaca, digestiva e mista. 0 estudo feito nos três períodos, supramencionados, permitiu ajuizar a evolução da doença de Chagas em cada indivíduo, sendo adotado os critérios de evolução: progressiva, inalterada e regressiva ${ }^{20}$.

\section{RESULTAD0S}

Foram estudados, parasitologicamente, 202 indivíduos, mas somente 190 fizeram 0 estudo clínico e parasitológico. A distribuição dos níveis de parasitemia dos 190 pacientes em 1976/78 foi: alta em 17, média em 38 e baixa em 135. Os níveis de parasitemia modificaram ao longo dos anos, de modo que em 1988/91 foi: alta em 8, média em 24 e baixa em 158, indivíduos 9 .

0 estudo eletrocardiográfico longitudinal foi realizado entre 1975 e 1991, teve duração média de 12,5 anos com ECG tomados em 1975/76, no interstício e em 1986/91. Dos 190, 110 (57,9\%) permaneceram com 0 exame normal, 23 (12,1\%) apresentaram alterações eletrocardiográficas, $13(6,8 \%)$ que tinham eletrocardiogramas alterados, normalizaram ou melhoraram 0 exame e 44 (23,3\%) permaneceram com 0 exame alterado ou desenvolveram mudanças mais graves ${ }^{10}$.

0 tempo médio de estudo radiológico do esôfago foi 13,3 anos. Foram realizadas 168 abreugrafias em 1975/76 e 187 em 1988/91 além dos exames no interstício. Somente 166 pacientes fizeram o exame em 1975/76 e repetiram em 1988/91. № esofagograma inicial de 1975/76, 142 (85,5\%) tiveram o exame normal, $12(7,2 \%)$ apresentaram esofagogramas duvidosos e $12(7,2 \%)$ apresentaram megaesôfago. № exame realizado em 1988/91, $119(71,7 \%)$ apresentaram resultados normais, 12 $(7,2 \%)$ tiveram esofagogramas duvidosos e $35(21,1 \%)$ apresentaram megaesôfago.

Seis chagásicos apresentaram obstipação intestinal de seis dias ou mais em 1975/76 e 18 indivíduos constipação maior de seis dias, em 1986/91. Apesar dos indivíduos apresentarem este sintoma em várias ocasiões, esta sintomatologia não foi constante, havendo períodos de melhora nesse intervalo de tempo. 
0 exame clínico, a obstipação, o ECG e 0 esofagograma de cada paciente permitiram concluir no estudo longitudinal, que $72(37,9 \%)$ chagásicos evoluíram de forma progressiva, $108(56,8 \%)$ permaneceram com a forma inalterada e $10(5,3 \%)$ regrediram as alterações eletrocardiográficas inespecíficas e não tinham alterações digestivas ${ }^{10}$.

Evolução da doença de Chagas e resultados dos xenodiagnósticos. Comparando 56 pacientes que tiveram pelo menos um xeno positivo e 134 que apresentaram os xenos negativos, no último exame feito em 1988/91, com os respectivos critérios de evolução, observou-se que houve evolução progressiva de $22(39,3 \%)$ e $50(37,3 \%)$ chagásicos xenopositivos e xenonegativos, respectivamente. A evolução inalterada permaneceu em $32(57,1 \%)$ e $76(56,7 \%)$ pessoas, xenopositivas e xenonegativas, respectivamente. Apenas a evolução regressiva tendeu a ser maior entre os xenonegativos, mas sem significância estatística ( Tabela 1).

Tabela 1-Distribuição de 190 indivíduosxenopositivosexenonegativos em relação à evolução clínica da doença de Chagas em 13 anos.

\begin{tabular}{|c|c|c|c|c|c|c|}
\hline \multirow{3}{*}{$\begin{array}{l}\text { Chagásicos } \\
1988 / 91\end{array}$} & \multicolumn{6}{|c|}{ Critérios de evolução } \\
\hline & \multicolumn{2}{|c|}{ progressiva } & \multicolumn{2}{|c|}{ inalterada } & \multicolumn{2}{|c|}{ regressiva } \\
\hline & $\mathrm{n}^{0}$ & $\%$ & $\mathrm{n}^{\underline{0}}$ & $\%$ & $\mathrm{n}^{0}$ & $\%$ \\
\hline Xenopositivos (56) & 22 & 39,3 & 32 & 57,1 & 2 & 3,6 \\
\hline Xenonegativos (134) & 50 & 37,3 & 76 & 56,7 & 8 & 6,0 \\
\hline Total (190) & 72 & 37,9 & 108 & 56,8 & 10 & 5,2 \\
\hline
\end{tabular}

Evolução da doença em relação aos níveis de parasitemia. Considerando-se inicialmente os níveis de parasitemia de 1976/78, em relação à evolução da doença, 13 anos após, observa-se que $3(17,6 \%)$ chagásicos de alta parasitemia, $15(39,5 \%)$ de média e $54(40 \%)$ de baixa parasitemia, apresentaram evolução progressiva, não havendo diferença estatística significante $(p>0,05)$ entre os três níveis de parasitemia, (Tabela 2). Considerando-se ainda os níveis de

Tabela 2 - Distribuição de 190 chagásicos com parasitemia de 1976/ 78, em relação à evolução clínica da doença de Chagas após 13 anos.

\begin{tabular}{|c|c|c|c|c|c|c|}
\hline \multirow{3}{*}{$\begin{array}{l}\text { Parasitemia } \\
1976 / 78\end{array}$} & \multicolumn{6}{|c|}{ Critérios de evolução } \\
\hline & \multicolumn{2}{|c|}{ progressiva } & \multicolumn{2}{|c|}{ inalterada } & \multicolumn{2}{|c|}{ regressiva } \\
\hline & $\mathrm{n}^{\underline{0}}$ & $\%$ & $\mathrm{n}^{0}$ & $\%$ & $\mathrm{n}^{0}$ & $\%$ \\
\hline Alta (17) & 3 & 17,6 & 13 & 76,5 & 1 & 5,9 \\
\hline Média (38) & 15 & 39,5 & 20 & 52,6 & 3 & 7,9 \\
\hline Baixa (135) & 54 & 40,0 & 75 & 55,6 & 6 & 4,4 \\
\hline Total (190) & 72 & 37,9 & 108 & 56,8 & 10 & 5,3 \\
\hline
\end{tabular}

Teste de Fisher em várias combinações $p>0,05$

parasitemia de 1988/91, em relação à evolução da doença, observa-se que $5(62,5 \%)$ chagásicos de alta parasitemia, 10 $(41,7 \%)$ de média e $57(36,1 \%)$ de baixa parasitemia apresentaram evolução progressiva, não havendo diferença estatística significante $(p>0,05)$ entre os três níveis de parasitemia, (Tabela 3). Porém, chama a atenção que quanto mais alta a parasitemia, maior foi o percentual dos que apresentaram evolução progressiva ocorrendo 0 contrário quando foi mais baixa a parasitemia. Na mesma tabela, observam-se os percentuais de chagásicos dos três níveis de parasitemia que apresentaram evolução inalterada e regressiva, não havendo também diferença estatística significante (Tabela 3). Se aos oito indivíduos de alta parasitemia acrescentarmos, cinco de média parasitemia que tiveram $50 \%$ ou mais dos pools positivos em 1988/91, haveria então 13 chagásicos com parasitemia elevada dos quais $7(53,8 \%)$ tiveram evolução progressiva e 6 (46,2\%) permaneceram com evolução inalterada. Nessa situação, também não há diferença estatística significante $(p>0,05)$ entre 0 s diversos níveis de parasitemia.

Evolução da doença nos pacientes sempre xenopositivos e xenonegativos. Houve 20 indivíduos que durante os 13 anos de estudo apresentaram todos os quatro xenos positivos e 59 que mostraram todos os quatro xenos negativos (Tabela 4). Observa-se que 6 (30\%) pessoas entre os xenopositivos e 17 (28,8\%) entre os xenonegtivos tiveram evolução progressiva. A evolução inalterada e regressiva foi percentualmente idêntica entre os xenopositivos e xenonegativos.

Tabela 3 - Distribuição de 190 chagásicos com parasitemia de 1988/ 91, em relação à evolução clínica da doença de Chagas.

\begin{tabular}{|c|c|c|c|c|c|c|}
\hline \multirow{3}{*}{$\begin{array}{l}\text { Parasitemia } \\
1988 / 91\end{array}$} & \multicolumn{6}{|c|}{ Critérios de evolução } \\
\hline & \multicolumn{2}{|c|}{ progressiva } & \multicolumn{2}{|c|}{ inalterada } & \multicolumn{2}{|c|}{ regressiva } \\
\hline & $n^{0}$ & $\%$ & $n^{0}$ & $\%$ & $\mathrm{n}^{0}$ & $\%$ \\
\hline$\overline{A l t a}(8)$ & 5 & 62,5 & 3 & 37,5 & 0 & - \\
\hline Média (24) & 10 & 41,7 & 13 & 54,2 & 1 & 4,2 \\
\hline Baixa (158) & 57 & 36,1 & 92 & 58,2 & 9 & 5,7 \\
\hline Total (190) & 72 & 37,9 & 108 & 56,8 & 10 & 5,3 \\
\hline
\end{tabular}

teste de Fisher em várias combinações $p>0,05$

Tabela 4 - Distribuição dos chagásicos que durante 13 anos foram sempre xenopositivos e sempre xenonegativos em relação à evolução clínica da doença.

\begin{tabular}{|c|c|c|c|c|c|c|}
\hline \multirow{3}{*}{$\begin{array}{l}\text { Parasitemia } \\
1976 / 91\end{array}$} & \multicolumn{6}{|c|}{ Critérios de evolução } \\
\hline & \multicolumn{2}{|c|}{ progressiva } & \multicolumn{2}{|c|}{ inalterada } & \multicolumn{2}{|c|}{ regressiva } \\
\hline & $\mathrm{n}^{0}$ & $\%$ & $\mathrm{n}^{0}$ & $\%$ & $\mathrm{n}^{0}$ & $\%$ \\
\hline Positiva (20) & 6 & 30,0 & 13 & 65,0 & 1 & 5,0 \\
\hline Negativa (59) & 17 & 28,8 & 39 & 66,1 & 3 & 5,0 \\
\hline Total (79) & 23 & 29,1 & 52 & 65,8 & 4 & 5,0 \\
\hline
\end{tabular}

teste de Fisher em várias combinações $p>0,05$

Evolução da doença nos pacientes com baixa parasitemia. No último xeno feito em 1988/91, houve 158 chagásicos de baixa parasitemia dos quais 24 foram xenopositivos e 134 xenonegativos (Tabela 5). Observa-se que entre os positivos e negativos, houve $7(29,2 \%)$ e $50(37,3 \%)$ indivíduos, respectivamente, com evolução progressiva enquanto 16 (66,6\%) e 76 (56,7\%) permaneceram com evolução inalterada. Também, em relação à evolução regressiva, não houve diferença estatística significante entre positivos e negativos.

Tabela 5 - Distribuição de 158 chagásicos de baixa parasitemia, xenopositivos exenonegativos, em relação a evolução dínica da doença.

\begin{tabular}{|c|c|c|c|c|c|c|}
\hline \multirow{3}{*}{$\begin{array}{l}\text { Parasitemia } \\
1988 / 91\end{array}$} & \multicolumn{6}{|c|}{ Critérios de evolução } \\
\hline & \multicolumn{2}{|c|}{ progressiva } & \multicolumn{2}{|c|}{ inalterada } & \multicolumn{2}{|c|}{ regressiva } \\
\hline & $\mathrm{n}^{-0}$ & $\%$ & $\mathrm{n}^{0}$ & $\%$ & $\mathrm{n}^{0}$ & $\%$ \\
\hline Baixa positiva (24) & 7 & 29,2 & 16 & 66,6 & 1 & 4,2 \\
\hline Baixa negativa (134) & 50 & 37,3 & 76 & 56,7 & 8 & 6,0 \\
\hline Total (158) & 57 & 36,1 & 92 & 58,2 & 9 & 5,7 \\
\hline
\end{tabular}

teste de Fisher em várias combinações $\mathrm{p}>0,05$ 
Evolução da doença nos pacientes com alta parasitemia e naqueles sempre xenonegativos. Analisando seis chagásicos que sempre apresentaram alta parasitemia e 59 que apresentaram todos os xenos negativos, durante os 13 anos de estudo, verificou-se que $3(50 \%)$ de alta parasitemia e $17(28,8 \%)$ sem parasitemia nos quatro xenodiagnósticos, respectivamente, apresentaram evolução progressiva (Tabela 6). Mesmo com este amplo gradiente percentual, não houve diferença estatisticamente significante $(p>0,05)$ pelo teste exato de Fisher, embora houve maior tendência para evolução progressiva daqueles com alta parasitemia.

Tabela 6 - Distribuição dos indivíduos que durante 13 anos sempre apresentaram alta parasitemia e daqueles com todos os xenos negativos, em relação à evolução clínica da doença.

\begin{tabular}{|c|c|c|c|c|c|c|}
\hline \multirow{3}{*}{$\begin{array}{l}\text { Parasitemia } \\
1976 / 91\end{array}$} & \multicolumn{6}{|c|}{ Critérios de evolução } \\
\hline & \multicolumn{2}{|c|}{ progressiva } & \multicolumn{2}{|c|}{ inalterada } & \multicolumn{2}{|c|}{ regressiva } \\
\hline & $\mathrm{n}^{0}$ & $\%$ & $\mathrm{n}^{0}$ & $\%$ & $\mathrm{n}^{0}$ & $\%$ \\
\hline Alta (6) & 3 & 50,0 & 3 & 50,0 & 0 & - \\
\hline Negativa (59) & 17 & 28,8 & 39 & 66,1 & 3 & 5,0 \\
\hline Total (65) & 20 & 32,8 & 42 & 62,5 & 3 & 4,5 \\
\hline
\end{tabular}

teste de Fisher em várias combinações $p>0,05$

Correlação entre média das idades, níveis de parasitemia e evolução da doença. Adistribuição das médias das idades dos 190 indivíduos estudados pode ser observada em relação aos níveis de parasitemia e às formas de evolução da doença ( Tabela 7) . A análise da média das idades dos indivíduos de alta, média e baixa parasitemias, não apresentou diferença estatística significante $(p>0,05)$. Por outro lado, a média das idades

Tabela 7 - Distribuição das médias das idades, de 190 chagásicos de vários níveis de parasitemia, em relação à evolução da doença.

\begin{tabular}{lcccc}
\hline Parasitemia & \multicolumn{3}{c}{ Médias das idades (anos) das } & média \\
& \multicolumn{3}{c}{ formas de evolução } & das idades \\
\cline { 2 - 4 } & progressiva & inalterada & regressiva & \\
\hline Alta & 45,8 & 29,3 & - & 39,6 \\
\multirow{4}{*}{ Média } & $(5)$ & $(3)$ & & $(8)$ \\
& 51,2 & 39,4 & 62,0 & 45,3 \\
Baixa & $(10)$ & $(13)$ & $(1)$ & $(24)$ \\
& 45,5 & 40,1 & 29,3 & 41,5 \\
& $(57)$ & $(92)$ & $(9)$ & $(158)$ \\
\hline Média das idades & $46,4 *$ & 39,8 & $32,6 *$ & 41,6 \\
& $(72)$ & $(108)$ & $(10)$ & $(190)$ \\
\hline
\end{tabular}

()$=\mathrm{n}^{0}$ de pacientes, análise de variância $* \mathrm{p}<0,05$

dos pacientes com evolução progressiva foi maior que a dos chagásicos com evolução regressiva, $p<0,05$. Dos 190 indivíduos estudados, houve 55 com 15 a 30 anos, média de idade 22,8 anos e 135 com 31 a 84 anos, média de idade 49,9 anos, cuja evolução progressiva foi, respectivamente, $14(25,4 \%)$ e 58 (43\%), não havendo diferença estatística significante $(\mathrm{p}>0,05)$.

\section{DISCUSSÃ0}

0 projeto Mambaí realiza estudo de coorte sobre a doença de Chagas desde 1975 e tem produzido dados clínicos e parasitológicos que podem contribuir para a discussão sobre 0 papel da parasitemia na evolução da doença crônica.

Há poucos estudos longitudinais da parasitemia nessa doença e todos utilizaram 0 xenodiagnóstico clássico. Dentre eles destaca-se 0 de Freitas ${ }^{15}$ que realizou até 11 xenodiagnósticos por paciente mas na maioria dos indivíduos investigados o tempo de estudo foi apenas de poucos meses. Estudos de parasitemia mais prolongados surgiram a partir da década de setenta ${ }^{11} \mathrm{e}$ mesmo assim há poucos trabalhos publicados, sendo o tempo máximo de estudo, oito anos ${ }^{21}$.

Neste trabalho, foi estudada a parasitemia dos pacientes por um período médio de 13 anos, tempo longo em relação os estudos similares existentes. Foi mostrado que a parasitemia de $8(4,2 \%)$ chagásicos permaneceu alta ou elevou-se ao longo dos anos, mesmo após a extinção do T. infestans dos domicílios e 20 chagásicos apresentaram os quatro xenos positivos durante os 13 anos de estudo. Considerando que a infecção chagásica ocorre em geral na infância e na adolescência e que 12 pacientes tinham 40 a 78 anos, supõe-se que alguns indivíduos permanecem toda a vida com parasitemia patente. Entretanto o que ocorreu com a maioria dos indivíduos do estudo foi o declínio da parasitemia.

0 papel da parasitemia na evolução da doença de Chagas tem sido discutido pelos pesquisadores ${ }^{1} 81112222327$. Há trabalhos que creditam à parasitemia influência no curso clínico da infecção ${ }^{12}{ }^{23}$, embora outros neguem sua participação na evolução da doença ${ }^{19}$.

Há trabalhos na literatura que mencionam a influência da parasitemia sobre a clínica da doença de Chagas crônica embora a maioria trata 0 tema de forma superficial. Cerisola et a ${ }^{11}$, tentando esclarecer 0 assunto, fizeram estudo clínico, eletrocardiográfico e parasitológico mensal em 30 pacientes durante 3 anos. Descobriram pacientes com diversos níveis de parasitemia porém nenhuma relação aparente entre as escassas lesões encontradas e 0 nível de parasitemia. Castro et al ${ }^{8}$, em estudo seccional de 292 pacientes, concluíram que a alta parasitemia não agrava a doença de Chagas crônica e detectaram que $74 \%$ dos indivíduos com alta parasitemia estavam na forma indeterminada. Schenone etal ${ }^{27}$ encontraram maior porcentagem de xenos positivos em chagásicos assintomáticos em relação àqueles com megaesôfago e cardiopatia. Ainda Schenone e Rojas ${ }^{28}$ realizando xenodiagnóstico em nove pacientes chagásicos crônicos, por um período médio de 3,3 anos verificaram que todos permaneceram na forma indeterminada apesar de terem níveis diferentes de parasitemia. Rassi et al ${ }^{25}$ verificaram que 0 aumento da parasitemia em chagásicos que usaram corticóides não influenciou a clínica da enfermidade. Pereira et $\mathrm{a}^{21}$ constataram em estudo de oito anos que a progressão da cardiopatia chagásica crônica não está associada ao xenodiagnóstico positivo embora, esses mesmos autores ${ }^{22}$, encontraram parasitemias mais altas em pacientes cardiopatas. Silva et $\mathrm{al}^{29}$ verificaram que a positividade do xenodiagnóstico mostrou-se independente da forma clínica da doença de Chagas. Ao contrário dos autores precedentes, Coura ${ }^{12}$ encontrou maior porcentagem de xenos positivos em indivíduos com cardiopatia e megaesôfago comparativamente àqueles somente com 
sorologia positiva e Pífano $0^{23}$, na Venezuela compartilha a mesma opinião. Adad ${ }^{1}$ estudando a patologia do megaesôfago chagásico, encontrou 0 T. cruzi em $50 \%$ dos megaesôfagos e em nenhum esôfago normal dos indivíduos infectados. Em estudo experimental, Miles et a $1^{18}$ trabalhando com macacos rhesus xenopositivos por mais de seis anos, não encontraram alterações eletrocardiográficas típicas da doença, nem correlação entre a parasitemia e parasitismo tecidual ao estudo anatomopatológico.

No presente trabalho, os pacientes que apresentaram reversão das alterações eletrocardiográficas eram mais jovens, tinham em média de 32,6 anos e nove deles apresentavam no início do estudo clínico em 1975/76 apenas bloqueio atrioventricular de primeiro grau.

Quanto à evolução da esofagopatia, metade dos pacientes que no início do estudo em 1975/76 tinham esofagogramas duvidosos desenvolveu megaesôfago e a maioria dos que já tinham megaesôfago evoluiu para esofagopatia mais grave. A incidência de megaesôfago nesse período foi 14,9\% (23/154), considerada alta quando comparada aos achados de Coura et a ${ }^{13}$, Macêdo ${ }^{17}$, Pereira eta ${ }^{20}$ e Pompeu ${ }^{24}$, porém inferior aos 18,3\% encontrados por Dias ${ }^{14}$ nos pacientes do estudo longitudinal em Bambuí, que tinham fase aguda conhecida.

Esse estudo sugere que a parasitemia nos diversos níveis, não tem influência na evolução da doença de Chagas crônica. № início do estudo, em 1976/78, 20\% (38) dos pacientes tinham parasitemia média e 9\% (17) parasitemia alta e ao longo do tempo ambos os percentuais decresceram. Apenas 4,2\% (8) permaneceram com alta parasitemia ao final do estudo em 1988/91. Quando se considera os 20 pacientes com parasitemia e os 59 sem parasitemia nos quatro xenos feitos durante 13 anos, em relação à clínica e achados dos exames complementares, observa-se que a evolução progressiva, inalterada e regressiva foi idêntica.

Ainda quando se considera os seis pacientes que tiveram alta parasitemia nos quatro xenos do início ao final do estudo e os 59 sempre xenonegativos durante todo o estudo observa-se que a evolução da doença foi idêntica não havendo diferença estatística significante contudo, percentualmente, houve maior tendência daqueles com alta parasitemia para evolução progressiva em relação aos xenonegativos. Infelizmente, houve poucos pacientes com alta parasitemia no material estudado.

A parasitemia e a evolução da doença de Chagas apresentam uma dinâmica temporal. Dos 190 pacientes desse trabalho, 17 tinham alta parasitemia em 1976/78, mas somente seis permaneceram com a mesma parasitemia 13 anos após e apenas três tiveram evolução progressiva, suas idades eram 24, 46 e 68 anos. Além dos seis já mencionados, que permaneceram com alta parasitemia, dois outros que tinham níveis de parasitemia menores, evoluíram para alta parasitemia em 1988/91. Ambos apresentaram evolução progressiva e suas idades eram 37 e 61 anos. De modo que em 1988/91 houve cinco pacientes de alta parasitemia que tiveram evolução progressiva.

Em conclusão, no Projeto Mambaí, tanto no estudo seccional $^{8}$ como no presente estudo, de 13 anos, não foi encontrada relação entre o nível de parasitemia e a evolução da doença de Chagas na fase crônica.

\section{REFERÊNCIAS BIBLIOGRÁFICAS}

1. Adad SJ. Contribuição ao estudo da anatomia patológica e da patogênese do megaesôfago chagásico.Tese de mestrado, Faculdade de Medicina do Triângulo Mineiro, Uberaba, Minas Gerais, 1989.

2. Almeida HO. Cardiopatia e parasitismo no chagásico crônico. Revista da Sociedade Brasileira de Medicina Tropical 17:157-159, 1984.

3. Almeida HO, Martins E, Franciscon JU, Teixeira VPA, Barbosa AJA, Gobbi H, Reis MA. Características das células parasitadas pelo Trypanosoma cruzi na parede da veia central das supra-renais de chagásicos crônicos. Revista da Sociedade Brasileira de Medicina Tropical 19:227-231, 1986.

4. Almeida HO, Teixeira VPA, Gobbi H, Rocha A, Brandão MC. Inflamação associada a células musculares cardíacas parasitadas pelo Trypanosoma cruzi, em chagásicos crônicos. Arquivos Brasileiros de Cardiologia 42:183186, 1984.

5. Almeida HO, Teixeira VPA, Oliveira ACA. Flebite com parasitismo em suprarenais de chagásicos crônicos. Arquivos Brasileiros de Cardiologia 36:341344, 1981.

6. Almeida HO, Teixeira VPA, Reis MA, Franciscon JU, Martins E. Modificações nucleares em células parasitadas pelo Trypanosoma cruzi em chagásicos crônicos. Revista da Sociedade Brasileira de Medicina Tropical 20:147. 152,1987.

7. Barbosa Jr AA, Andrade ZA. Identificação do Trypanosoma cruzi nos tecidos extracardíacos de portadores de miocardite crônica chagásica. Revista da Sociedade Brasileira de Medicina Tropical 17:123-126,1984.

8. Castro C. Influência da parasitemia no quadro clínico da doença de Chagas. Revista de Patologia Tropical 9: 73-136,1980.

9. Castro C, Macêdo V, Prata A. Comportamento da parasitemia pelo Trypanosoma cruzi em chagásicos crônicos durante 13 anos. Revista da Sociedade Brasileira de Medicina Tropical 32:157-165, 1999.

10. Castro C, Prata A, Macêdo V. Estudo clínico durante 13 anos de 190 chagásicos crônicos de Mambaí, Goiás, Brasil. Revista da Sociedade Brasileira de Medicina Tropical 34:309-318, 2001.

11. Cerisola JA, Rohwedder R, Segura EL, Del Prado CE, Alvarez M, De Martini GJW. El xenodiagnostico. Ministério de Bienestar Social, Buenos Aires, Argentina, p 84, 1974

12. Coura JR. Evolutive pattern in Chagas' disease and the life span os Trypanosoma cruzi in human infection. American Trypanosomiase Research, Belo Horizonte, p 378-386, 1975.

13. Coura JR, Abreu LL, Pereira JB, Willcox HP. Morbidade da doença de Chagas. IV. Estudo longitudinal de 10 anos em Pains e Iguatama, Minas Gerais, Brasil. Memórias do Instituto Oswaldo Cruz 80: 73-80, 1985.

14. Dias JCP. Doença de Chagas em Bambuí, Minas Gerais, Brasil. Estudo clínico epidemiológico a partir da fase aguda, entre 1940-1982. Tese de Doutorado, Universidade Federal de Minas Gerais, Belo Horizonte, 1982.

15. Freitas JLP. Contribuição para 0 estudo do diagnóstico da molestia de Chagas por processos de laboratório. Tese de livre docência, Faculdade de Medicina da Universidade de São Paulo, São Paulo, 1947.

16. Gobbi H, Teixeira VPA, Fernandes PA, Brandão MC, Reis MA, Almeida HO. Parasitismo incomum na veia central da supra-renal em chagásicos crônicos. Revista da Sociedade Brasileira de Medicina Tropical 19 ( supl II) : 36, 1986.

17. Macêdo V. Influência da exposição à reinfecção na evolução da doença de Chagas. Revista de Patologia Tropical 5:33-116,1976.

18. Miles MA, Marsden PD, Pettitt LE, Drapper CC, Sarah W, Seah SKK. Experimental Trypanosoma cruzi infection in rhesus monkeys. III eletrocardiographic and histopatological findings. Transaction of the Royal Society of Tropical Medicine and Hygiene 73:528-532, 1979.

19. Pereira JB, Coura JR. Evolução eletrocardiográfica na doença de Chagas crônica humana. I - Influência da parasitemia. Revista da Sociedade Brasileira de Medicina Tropical 24 (supl I) : 26-27, 1991.

20. Pereira JB, Willcox HP, Coura JR. Morbidade da doença de Chagas. III. Estudo longitudinal de seis anos, em Virgem da Lapa, MG, Brasil. Memórias do Instituto Oswaldo Cruz 80: 63-71,1985. 
21. Pereira JB, Willcox HP, Coura JR. Evolução da cardiopatia chagásica crônica I. Influência da parasitemia. Revista da Sociedade Brasileira de Medicina Tropical 25:101-108, 1992.

22. Pereira JB, Willcox HPF, Marcondes CB, Coura JR. Parasitemia em pacientes chagásicos crônicos, avaliada pelo índice de triatomíneos infectados no xenodiagnóstico. Revista da Sociedade Brasileira de Medicina Tropical 22:39-44, 1989.

23. Pífano CF. La miocardiopatia chagásica crônica en el médio rural venezolano. Gaceta Medica de Caracas 85:17-30, 1977.

24. Pompeu FR. Estudo longitudinal da doença de Chagas em trabalhadores rurais do Município de Luz, Minas Gerais (1976-1985) , Tese de mestrado, Universidade Federal de Minas Gerais, Belo Horizonte, 1990.

25. Rassi A, Amato Neto V, Siqueira AF, Doles J, Leite MSB, Silva OQ, Cardoso VM. Influência de corticoide, na doença de Chagas crônica, administrado em virtude de afecçoes associadas. Revista da Sociedade Brasileira de Medicina Tropical 30:93-99, 1997.

26. Rezende JM, Lauar KM, Oliveira AR. Aspéctos clínicos e radiológicos da aperistalse do esôfago. Revista Brasileira de Gastroenterologia 12:247. $262,1960$.
27. Schenone H, Alfaro E, Reyes H, Taucher E. Valor del xenodiagnostico en la infeccion chagásica crônica. Boletin Chileno de Parasitologia 23: 49-154, 1968.

28. Schenone H, Rojas S. Estudio longitudinal mediante el xenodiagnostico de la parasitemia en pacientes com infeccion cronica pelo T. cruzi. Boletin Chileno de Parasitologia 54:29-32, 1999.

29. Silva IG, Silva HHG, Ostermayer AL, Rezende JM. Positividade do xenodiagnóstico de acordo com a faixa etária, sexo e a forma clínica da doença de Chagas. Revista de Patologia Tropical 24:193-197, 1995.

30. Teixeira VPA, Almeida HO. Parasitismo da veia central da supra-renal em diferentes formas anátomo-clínicas da doença de Chagas. Revista da Sociedade Brasileira de Medicina Tropical 19 ( Supl II) 35, 1986

31. Teixeira VPA, Fernandes PA, Brandão MC, Gobbi H, Reis MA, Almeida HO. Parasitismo incomum na veia central da supra-renal em chagásicos crônicos. Arquivos Brasileiros de Cardiologia 47:425-428, 1986.

32. Teixeira VPA, Reis MA, Araújo MBM, Silveira AS, Reis L, Almeida HO. Comparação do parasitismo da veia central da supra-renal com o de outros tecidos em chagásicos crônicos. Revista da Sociedade Brasileira de Medicina Tropical 24:73-78, 1991. 